\title{
Por una geopolítica de las lenguas romances*
}

\section{Louis-Jean Calvet}

Traducción del francés al español de Jessica Montaño Oliveros Universidad de Antioquia, Medellín, Colombia jmontanooliveros@gmail.com

\section{RESUMEN}

Este artículo de reflexión nos propone un breve recorrido histórico de la política contemporánea de las lenguas romances y su relación con los demás grupos lingüísticos (xfonías). A través de una analogía con la teoría de juegos, establecida alrededor de los diferentes organismos e instituciones propias de cada región, se evidencia la influencia de los intereses de la globalización a nivel cultural y, sobre todo, económico; el discurso de lo políticamente correcto limita las iniciativas para una intercomprensión entre la hispanofonía, la francofonía y la lusofonía. Sin embargo, existe una solución viable desde el punto de vista educativo con el fin de mejorar el futuro de la geopolítica lingüística romance.

Palabras clave: geopolítica; lenguas romances; teoría de juegos; intercomprensión

\section{Introducción}

Quisiera comenzar por referir una constatación simple: nos encontramos en un continente en el que dominan las lenguas romances. Si se mira desde lejos, desde Europa, por ejemplo, esa afirmación parece paradójica, pero veremos que esta es una realidad indiscutible.

Empecemos por la situación a nivel mundial de estas lenguas a partir de algunas consideraciones. Primeramente, cabe preguntarse ¿cuántas personas en todo el mundo tienen una lengua romance como lengua materna? En la tabla 1 se verá que las cifras que ofrece el sitio Ethnologue (Eberhard, Simons y Fenning, 2019) muestran que llegamos a ser un total de 766 millones de hablantes.

Cómo citar: Calvet, L.-J. (2021). Por una geopolítica de las lenguas romances (J. Montaño, trad.). Ciencias Sociales y Educación, 10(20), 285-297. https://doi.org/10.22395/csye.v10n20a13

Traducción realizada por Jessica Montaño Oliveros del artículo "Pour une géopolitique des langues romanes" de Calvet (2018).

Agradecemos a la revista Matices en Lenguas Extranjeras (MALE) de la Universidad Nacional de Colombia permitir la publicación del texto de Calvet en su versión en español en nuestra revista. Recibido: 8 de junio de 2021.

Aprobado: 20 de junio de 2021. 
Tabla 1.Número de hablantes por cada lengua tenida en cuenta como lengua materna según Ethnologue

\begin{tabular}{lc}
\hline Lengua & Número de hablantes (en millones) \\
\hline Catalán & 4 \\
Español & 398 \\
Francés & 75 \\
Italiano & 63 \\
Portugués & 203 \\
Rumano & 23 \\
\hline
\end{tabular}

Fuente: Eberhard, Simmons y Fenning (2019) ${ }^{1}$.

Otra manera de medir el peso o la importancia de una lengua es a partir del factor de uso. Así, se verá en la tabla 2 que el español es oficial en veintiún países, el francés en treinta y seis, etc.; lo que lleva a un total de setenta países en los que la lengua oficial o nacional es una lengua romance.

Tabla 2. Países donde el español, el francés, el italiano, el portugués o el rumano son oficiales

\begin{tabular}{lc}
\hline Lengua oficial & Número de países \\
\hline Español & 21 \\
Francés & 36 \\
Italiano & 3 \\
Portugués & 8 \\
Rumano & 2 \\
\hline
\end{tabular}

Fuente: National Encyclopédie Wikipedia (citado por Leclerc, 2017).

El total es de setenta países, es decir, más de un tercio de los países miembros de la ONU. En la figura 1 se verá un mapa de lenguas oficiales en el mundo, donde hay grandes manchas rosadas para el inglés, amarillas para el árabe, verdes para el chino, naranjas para el español, etc.; sin embargo —aparte de la India—, en el mapa no se tienen en cuenta las lenguas cooficiales. 


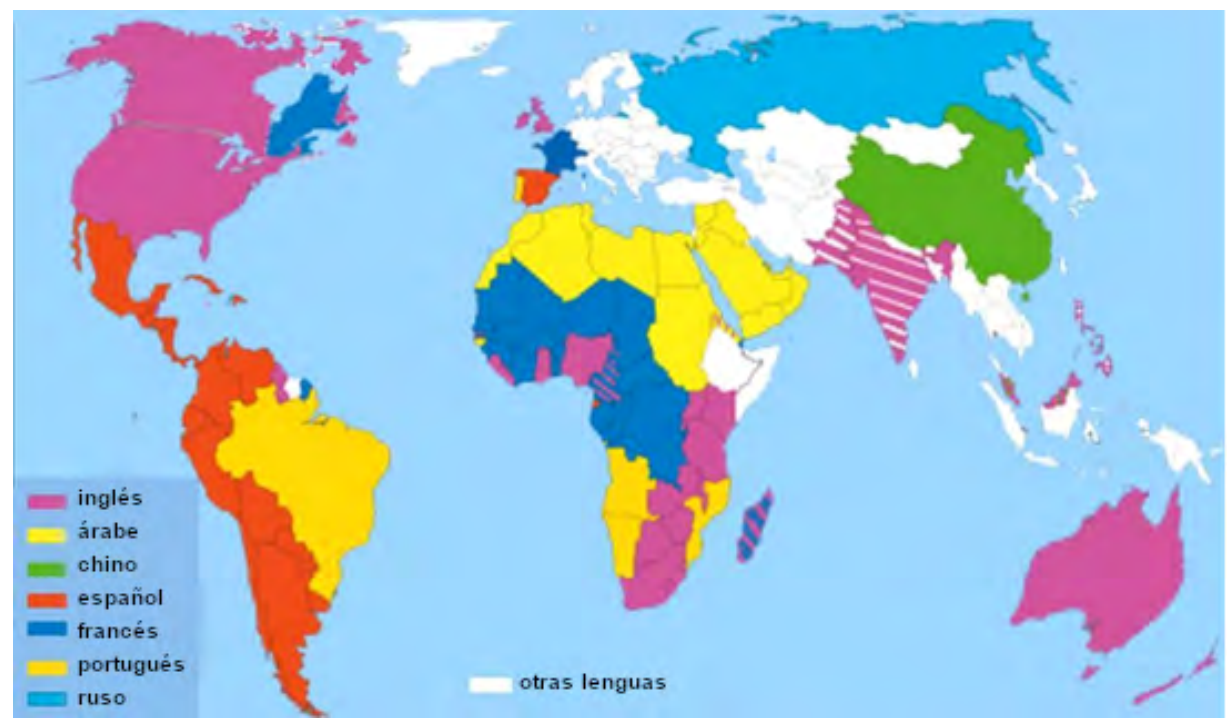

Figura 1. Lenguas con estatus de lenguas oficiales.

Fuente: Mapa tomado originalmente del informe de la Organización Internacional de la Francofonía (2006-2007) Français dans le Monde (citado y adaptado por RFI, 2007 [2009]).

Este otro mapa, que se verá en la figura 2, sí tiene en cuenta las lenguas cooficiales para Canadá; además, veremos que estas lenguas europeas, que vienen probablemente de la Cuenca del Mediterráneo, están presentes en Europa, en toda América y el Caribe y en África.

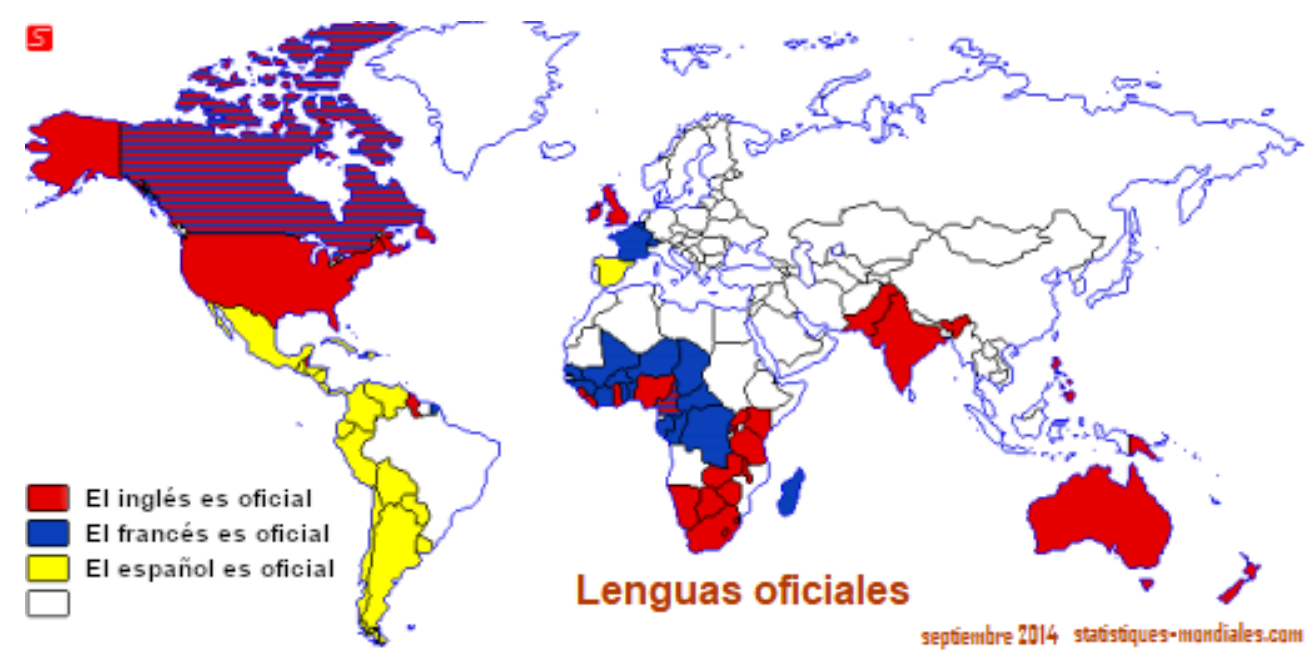

Figura 2. Lenguas oficiales en el mundo.

Fuente: Mapa tomado originalmente de Statistiques Mondiales (2014). 
A lo anterior debe agregarse que el $17 \%$ de los 325 millones de habitantes de Estados Unidos habla español. Y para terminar con estas consideraciones rápidas, tenemos la tabla 3 que muestra las primeras veinte lenguas del barómetro Calvet, entre las que se encuentran cuatro lenguas romances.

Tabla 3. Primeras lenguas del barómetro Calvet

\begin{tabular}{cc}
\hline 1. Inglés & 11. Mandarín \\
2. Francés & 12. Polaco \\
3. Español & 13. Checo \\
4. Alemán & 14. Croata \\
5. Ruso & 15. Rumano \\
6. Italiano & 16. Serbio \\
7. Portugués & 17. Húngaro \\
8. Japonés & 18. Coreano \\
9. Neerlandés & 19. Noruego \\
10. Sueco & 20. Danés \\
\hline
\end{tabular}

Fuente: wikiLF (2017).

Este barómetro tiene en cuenta once factores y aquí se añadirá uno más: las lenguas que se enseñan en las universidades como lenguas extranjeras. La tabla 4 indica la situación mundial de estas lenguas (podría cambiar según los continentes, por ejemplo, el chino y el japonés tienen más participación en Australia) (Calvet Y Calvet, 2016). Se puede ver que el inglés se enseña en el $97 \%$ de las universidades del mundo, sin contar las de los países anglófonos; que el francés se enseña en el $74 \%$ de las universidades, sin contar las de los países francófonos; etc.

Tabla 4. Lenguas extranjeras que se enseñan / \% de universidades

\begin{tabular}{llll}
\hline 1. Inglés & $97 \%$ & 7. Chino & $37,8 \%$ \\
2. Francés & $74,9 \%$ & 8. Japonés & $34,9 \%$ \\
3. Alemán & $62 \%$ & 9. Árabe & $28,7 \%$ \\
4. Español & $50,8 \%$ & 10. Portugués & $23,6 \%$ \\
5. Italiano & $40,8 \%$ & 11. Coreano & $14,6 \%$ \\
6. Ruso & $38,6 \%$ & 12. Turco & $12,4 \%$ \\
\hline
\end{tabular}

Fuente: Calvet (2016). 
Además, en la figura 3 se puede ver una representación más diciente de los mismos porcentajes.

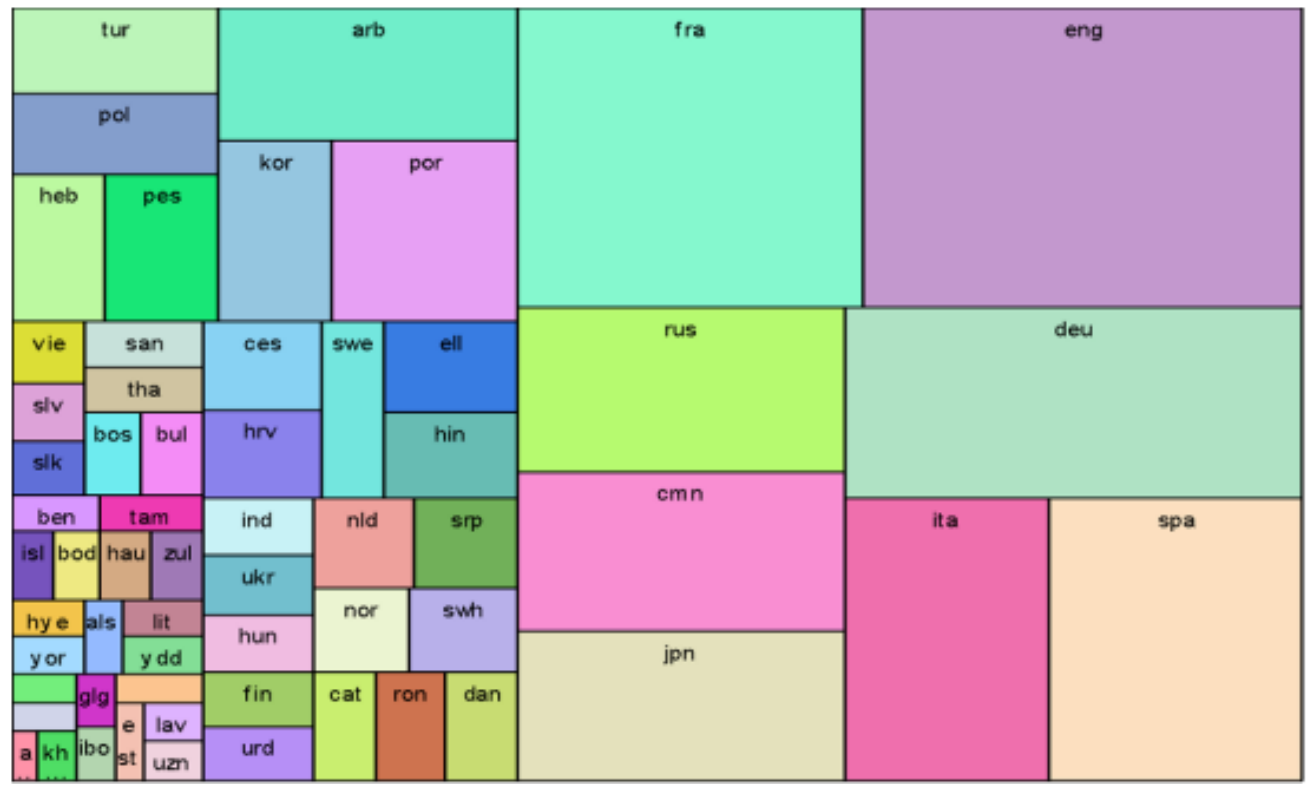

Figura 3. Diagrama del porcentaje de lenguas que se enseñan en las universidades

Fuente: Calvet (2018).

En la mitad derecha del diagrama se encuentran ocho lenguas, de las cuales seis tienen origen europeo; el árabe aparece en novena posición, pero, al igual que en la clasificación de lenguas oficiales, normalmente se refiere al árabe escrito.

Todo esto sirve para recordarnos que, desde diferentes aspectos, las lenguas romances gozan de una posición importante en el mundo. Además de estas consideraciones geolingüísticas y politicolingüísticas, se debe considerar también otro factor: la intercomprensión entre estas lenguas, tema que abordan Blanche-Benveniste y Valli (1997) en L'intercompréhension: le cas des langues romanes, Dabène (2003) y también aparece en un curso en línea abierto y masivo (CLOM) de intercomprensión de las lenguas romances de la Organización Internacional de la Francofonía (2015).

\section{Las lenguas y la teoría de juegos}

Ustedes dirán que todo lo anterior es muy bonito, ¿pero y qué? La francofonía, la hispanofonía y la lusofonía ciertamente existen de manera más o menos organizada, pero existen de manera independiente. El Instituto Cervantes, el 
Lovis-Jean Calvet

Instituto Camões y los Institutos Franceses o las Alianzas Francesas aplican sus propias políticas individuales que, a veces, pueden ser opuestas.

Y es justamente ahí que la teoría de juegos puede resultar útil. En esta teoría se dice que hay un problema de juego, porque existen varios centros de decisión. Ahora bien, sin importar si las políticas lingüísticas versan sobre varias lenguas en un mismo nicho ecolingüístico (como el francés, el corso, el bretón, etc.) o sobre varios espacios lingüísticos (como la francofonía, la hispanofonía, la anglofonía, etc.), le competen a diferentes responsables o agentes que podrían considerarse los "jugadores" en el sentido que se precisará más adelante. Retomemos los dos tipos de juego que menciona Calvet (2002):

En todas las situaciones (políticas, diplomáticas, militares, etc.) en las que los "jugadores" tienen intereses diferentes, se deben tener en cuenta dos factores, la cooperación y la competencia, que se conjugarán, por convergencia o divergencia de sus intereses, para dar lugar a juegos de cooperación, de competencia o de cooperación y competencia. En los juegos de cooperación, los intereses de los jugadores convergen frente a un único adversario, los jugadores pueden adoptar una estrategia común para lograr un fin común. Por el contrario, en los juegos de competencia no existen intereses comunes, los jugadores no tienen un fin común y se encuentran en una situación de contienda. Finalmente, en los juegos de competencia y cooperación, los jugadores tienen algunos intereses convergentes y otros divergentes ${ }^{2}$.

En tales situaciones, el jugador debe construir un modelo que represente la situación real, teniendo en cuenta las posibilidades que le ofrecen a él y las que se les ofrecen a los otros jugadores. En este modelo, tendrá que integrar las consecuencias de las decisiones que él mismo tomará con las que tomarán los otros jugadores. Para hacerlo, tendrá que considerar cuáles pueden ser sus preferencias y las de los demás, y si existen alianzas posibles, es decir, convergencias de preferencias que podrían llevar los intereses individuales de los jugadores hacia un interés colectivo. A estas consideraciones se añade además la distinción entre los juegos de suma cero y suma positiva. En el primer caso, lo que gana uno de los jugadores es igual a lo que pierden los demás, las ganancias de uno equivalen a las pérdidas del otro. Esto es lo que sucede, por ejemplo, en una partida de póker. En el segundo caso, todos ganan, pero no ganan lo mismo.

Pasemos ahora a las políticas lingüísticas, pues aquí la analogía con la teoría de juegos ofrece un criterio muy razonable:

Si consideráramos metafóricamente que los "jugadores" son las lenguas (es decir, el grupo de sus hablantes) o los grupos lingüísticos (francofonía, anglofonía, etc.), entonces se podría analizar su relación como un juego; el problema es saber en qué puntos puede haber cooperación y en qué puntos existe el riesgo de contienda. Se podrían analizar, por ejemplo, los problemas lingüísticos de Europa al intentar

Esta y todas las citas textuales subsiguientes son traducciones propias. Nota de la traductora. 
descubrir los intereses de cada lengua, las posibilidades ofrecidas y las posibles consecuencias de las diferentes elecciones. (Calvet, 2007)

Esta es más o menos la situación en la que se encontrarían algunos grupos lingüísticos si decidieran formar alianzas.

Retomando por última vez la teoría de juegos, cuando un jugador elige una estrategia, se le puede asociar un cierto número de resultados esperados según las estrategias de que dispongan los demás jugadores. Pero si la estrategia es una cooperación entre los otros jugadores o una parte de ellos, debería asociarse a la estrategia común cuyos objetivos no son necesariamente los mismos para todos y que a veces es cuestión de compromiso. Consideremos un ejemplo hipotético sobre este tema (Calvet, 2007):

Y si consideramos que la política lingüística no debería ser un juego de suma cero, es posible imaginar que cada jugador se beneficiará de dicha cooperación aunque no tengan el mismo objetivo, pero sí objetivos compatibles. Ninguna cooperación sería posible, por ejemplo, entre la francofonía y la hispanofonía si cada uno de estos dos grupos quisiera que su lengua fuera la segunda lengua internacional después del inglés.

Así, este enfoque implica que, paralelamente a un análisis concreto de la situación de las lenguas, que he comenzado a hacer anteriormente, debemos hacer un inventario de las expectativas, reivindicaciones y esperanzas de las diferentes lenguas o grupos lingüísticos; una lista de los problemas internos y externos que enfrentan a fin de identificar posibles concertaciones y acciones conjuntas entre todos o algunos de los jugadores en cuestión.

Para los grupos lingüísticos organizados propuse el término xfonías y el tema que quiero abordar ahora es el de posibles alianzas entre esas xfonías. Sin embargo, esta idea no es nueva: sobre ella existen algunas prefiguraciones que referiremos rápidamente.

La primera es la que se refiere al grupo de lenguas romances, que surgió en 1954: la Unión Latina. Este era un proyecto de alianza para la difusión de las lenguas (nacionales) de origen latino, es decir, el español, el francés, el italiano, el portugués y el rumano. Aunque esta iniciativa no tuvo mucho éxito durante los primeros años, el diplomático Philippe Rossillon la volvió a lanzar a principios de los ochenta, invirtiendo en ello su energía y sus propios recursos financieros. La Unión Latina se consagró, en esencia, a la terminología y dejó de funcionar en 2012, pero hay quienes quieren volverla a lanzar. Este es un ejemplo sobre el cual meditar. Esta estructura que se preocupó poco por la política lingüística (la terminología es solo una pequeña parte de la política lingüística) podría ser reavivada y usada para nuevos proyectos. El 7 de abril de 1997 en Lisboa, los treinta y cinco Estados miembros de esta unión firmaron 
una declaración (Conseil de l'Europe, 1997) en la que señalaban el peligro de un empobrecimiento cultural de la humanidad a partir de la unificación lingüística mundial, y claramente se preocuparon por hacerle contrapeso a la galopante transformación del mundo al anglicismo.

Pero a finales del siglo XX y principios del XXI las cosas cambiaron de curso. Hasta este punto y desde hacía treinta siglos, las lenguas de gran difusión apenas habían jugado un papel regional: desde el acadio en Mesopotamia hasta el francés en Europa, pasando por la koiné de Grecia, el latín o la lingua franca; algunos lugares del mundo conocieron lenguas vehiculares limitadas tanto regional como funcionalmente (según el caso, eran usadas por los comerciantes, los nobles, los científicos, los diplomáticos, etc.). Y por aquella época nadie se oponía a esas lenguas. Hoy la situación es diferente al menos por dos razones: el surgimiento cada vez más fuerte de nacionalismos y el miedo cada vez más palpable que suscita la globalización. El inglés ya no solo se considera una lengua imperial, también se percibe de manera errada como la traducción lingüística de un orden mundial que se critica desde diversos puntos de vista y por diversos motivos a través de diversas manifestaciones: los OGM, la OMC, el FMI, etc.

Pero la oposición a estas diversas manifestaciones de la globalización provenía principalmente de grupos de "centro", autoproclamados defensores de la periferia, y respecto a esto puede haber un gran malentendido. Efectivamente, mientras los ricos del centro protestan contra el maíz transgénico, por ejemplo, la gente de la periferia se muere de hambre; y los países del tercer mundo pueden ver en los OGM una solución a sus problemas. Lo mismo aplica en el caso de las lenguas. La francofonía, impulsada por Francia y Quebec, se ha movilizado para defender el francés, incorporando bajo esta bandera a los países africanos sin tener en cuenta los problemas lingüísticos propios de esos países. Solo recientemente la francofonía se ha planteado un nuevo tema: la necesaria diversidad que se ve amenazada por el inglés. Así, han redescubierto en el ámbito lingüístico una competencia que unos años antes era librada por la excepción cultural.

En este contexto nació la idea de la alianza entre las xfonías donde la francofonía (representada por la Organización Internacional de la Francofonía, OIF) acudió a otros dos grandes grupos lingüísticos: la hispanofonía y la lusofonía. Así, en el 2001, la OIF motivó varios encuentros destinados a "tres espacios lingüísticos" (la francofonía, la hispanofonía y la lusofonía) (Hoyos et al., 2001), siempre con énfasis en la "diversidad". Y, como se verá, esta distinción entre centro y periferia juega allí un rol importante.

¿Quiénes son sus asociados? Para empezar, una estructura un tanto peculiar en la medida en que no es completamente hispanófona, la Organización de Estados Iberoamericanos (OEI), cuya sede principal está en Madrid, con 
sucursales en Bogotá, Buenos Aires, Lima, México y San Salvador. Veintitrés países son miembros de esta organización: Argentina, Bolivia, Brasil, Colombia, Costa Rica, Cuba, Chile, República Dominicana, Ecuador, España, Guatemala, Guinea Ecuatorial, Honduras, México, Nicaragua, Panamá, Paraguay, Perú, Portugal, Puerto Rico, Salvador, Uruguay y Venezuela. Desde su creación, la OEI ha experimentado una evolución importante. Fue fundada en 1949 en Madrid, con ocasión del Primer Congreso Iberoamericano de Educación, pero con una función limitada que se refleja en su nombre de entonces: Oficina de Educación Iberoamericana. En 1985, en el congreso de Bogotá, esta "oficina" cambió su nombre y se convirtió en la Organización de Estados Iberoamericanos, con un subtítulo adicional que precisaba "para la Educación, la Ciencia y la Cultura", lo que la asemeja a una suerte de Unesco hispanolusófona. Después, en 1991, la OEI decidió organizar una Cumbre anual de jefes de Estado, preparada por una reunión de ministros de educación. Reunida cada cuatro años, la asamblea general de la OEI es la instancia legislativa que establece su política, aprueba su plan de operaciones, sus programas y elige al secretario general. La OEI tiene actualmente una estructura similar a la de la OIF, pero con la particularidad de ser bilingüe: junto a los veintiún miembros hispanófonos encontramos a dos países lusófonos: uno ibérico, Portugal; y uno americano, Brasil.

En cuanto a la lusofonía, esta se encuentra organizada en la Comunidad de Países de Lengua Portuguesa (CPLP), que fue creada el 17 de julio de 1996 durante la primera conferencia de jefes de Estado y de gobierno de los siguientes países: Angola, Brasil, Cabo Verde, Guinea-Bisáu, Mozambique, Portugal y Santo Tomé y Príncipe. Esta joven organización, cuya sede principal queda en Lisboa, tiene una estructura que parece inspirarse directamente en la de la OIF. Sus estatutos precisan en el artículo tercero que los países miembros sostienen una cooperación en términos económicos, sociales, culturales y jurídicos que trabajan por promover y difundir la lengua portuguesa por todo el mundo. A esto se debe añadir la existencia del Instituto Internacional de la Lengua Portuguesa (IILP), cuya sede principal queda en Praia (Cabo Verde) y cuya creación es muy reciente como para evaluar su producción. Sus principales objetivos son "la promoción, la defensa, el enriquecimiento y la difusión de la lengua portuguesa como vehículo para la cultura, la educación, la información y el acceso a conocimientos científicos y tecnológicos".

Durante dos o tres años, dos comisiones trabajaron en este proyecto de tres espacios, una sobre nuevas tecnologías y la otra sobre políticas lingüísticas. Yo presidí esta última. Allí insistimos mucho en el hecho de que, en cada una de las xfonías en cuestión, debía distinguirse entre la diversidad horizontal y la diversidad vertical. Una diversidad horizontal entre las tres lenguas dominantes: 
español, francés e inglés. Y, dentro de cada una de las xfonías, una diversidad vertical entre esa lengua y las otras. Por ejemplo, en la francofonía, la relación entre el francés y los idiomas africanos o los idiomas regionales. En la hispanofonía, la relación entre el castellano, las lenguas indígenas de América, el vasco, el catalán, etc. Y decíamos que si estos lenguajes periféricos no eran tenidos en cuenta, corríamos el riesgo de caer en una especie de reparto del mundo, una conferencia de Yalta lingüística para asegurar el estatus de estas tres lenguas con respecto al inglés.

Por lo tanto, hemos propuesto al mismo tiempo proyectos de políticas lingüísticas relativas a estas tres lenguas y proyectos relativos a la relación entre cada una de estas lenguas y sus lenguas "asociadas". Los Estados no nos siguieron en este segundo punto, quizás porque la francofonía se interesaba más en la defensa del francés que en la defensa de las lenguas que confluyen con él.

A diferencia de la francofonía, la hispanofonía no tiene que preocuparse por la difusión de su idioma en los países hispanófonos. De hecho, la mayoría de los países en los que el español es lengua oficial son hispanófonos (contrario a los que sucede en los países africanos "francófonos" o "anglófonos"). En el libro 1998 Britannica Book of the Year (Calhoun, 1998) se presentaban los siguientes porcentajes de ciudadanos que hablaban el idioma oficial de su país:

- inglés $27 \%$

- español $94,6 \%$

- francés $34,6 \%$

Por lo tanto, el español no está amenazado en los países hispanófonos y el problema de la defensa o promoción de esta lengua en el mundo no es un punto principal en la agenda. De hecho, hasta ahora, España ha estado mucho más preocupada por su expansión económica en los países hispanófonos que por la difusión de su lengua, que no necesitan transmitir allí. España aprovecha este espacio principalmente para extenderse económicamente: es el principal inversor en Argentina. Telefónica se estableció en Miami y compró el portal Lycos, la compañía petrolera Repsol compró a la argentina YPF, el Banco de Bilbao y Vizcaya (BBVA) tomó el control del mayor banco mexicano, etc. Además, España no es el principal país hispanófono: con unos cuarenta millones de habitantes está después de México (ochenta millones) y podría ser superada por Colombia y Argentina (unos treinta y cuatro y treinta y tres millones respectivamente), incluso por Estados Unidos, donde actualmente hay cerca de veintitrés millones de hispanófonos. 
Y la situación del portugués en la lusofonía es comparable a la del español en la hispanofonía: el número de lusófonos portugueses es casi diecisiete veces menor que el de lusófonos brasileños.

\section{Conclusión}

¿Tendría futuro entonces una cooperación entre las xfonías romances? ¿Y cuál es el futuro lingüístico del mundo? Para intentar dar respuesta a estas preguntas debemos saber cuál es el horizonte temporal sobre el que reflexionamos. Naturalmente, es imposible saber cuál será la situación dentro de mil años. Como decía Woody Allen, "es muy difícil prever algo, especialmente el futuro". A lo sumo podríamos suponer que ninguna de nuestras lenguas actuales será comprensible para los terrícolas, si todavía queda alguno. ¿Sería razonable pensar que en diez años la situación será la misma que tenemos actualmente? Sin duda, parecerá similar para aquellos que la viven sin analizarla. Sin embargo, todos los indicadores que presentamos aquí nos hacen pensar que las cosas habrán evolucionado: habrá muchas menos lenguas, muchos más hablantes para algunas lenguas y, quizás, unos cambios significativos en el estatus de otras lenguas. Entre esos dos horizontes temporales, diez años y mil años, ¿en dónde se encuentra nuestro futuro?

Si nuestro objetivo es tomar las reflexiones de la politología lingüística como base para unas políticas lingüísticas concretas, también debemos considerar cuál es el tiempo de la política, además de considerar lo que llamaré el tiempo de la lingüística o el tiempo de las lenguas. Normalmente, los políticos piensan a corto plazo: su horizonte está determinado por la siguiente elección, por el momento en que aspirarán una vez más al voto de los electores, con la esperanza de ganar. Sus decisiones se guían por el interés en obtener una rentabilidad inmediata o solo por el de hacerse visibles y producir un impacto. Pero las acciones con respecto a las lenguas y a las situaciones lingüísticas rara vez tienen repercusiones inmediatas: por cada intervención con resultados rápidamente visibles, como la de la "revolución lingüística" turca, hay muchas más que solo se pueden medir décadas después. Por lo tanto, es más fácil conseguir que los políticos hagan intervenciones lingüísticas simbólicas (casi siempre en forma de afirmaciones que no cuestan nada pero que no cambian mucho la situación); basta con adaptar elementos del discurso politicolingüísticamente correcto a alguna realidad, en lugar de realizar acciones con resultados a mediano o largo plazo.

Ahora, suponiendo que algunos de ellos se preocupen más por el futuro que por su reelección, ese futuro no puede estar muy lejos: para justificar una política, sus efectos deben ser como mínimo imaginables, si no palpables; es necesario que el ciudadano pueda pensar que si él no se beneficiará, al menos lo harán 
sus hijos o sus nietos. Se puede decir que la política es el arte de hacer posible aquello que se desea, pero ese deseo no puede ser muy lejano ni muy abstracto.

Pues bien, estas son algunas ideas, algunos proyectos que podríamos sacar adelante. Primero se podrían definir políticas lingüísticas dentro de cada país de lengua romance y después definir políticas lingüísticas de las lenguas romances a escala mundial frente a otras xfonías. Para poner un ejemplo simple, podríamos actuar frente a la lista de lenguas oficiales de las instituciones internacionales, proponiendo que se incluya el portugués, y también el alemán o el hindi o el swahili.

Pero tomemos un ejemplo que nos concierne directamente: la situación del francés en América. En América Latina y central, el inglés es obligatorio y el francés no es más que la segunda lengua que enseñan, aunque puede concurrir con el español en Brasil o con el portugués en Argentina, etc. Por consiguiente, ¿por qué no proponer la introducción de un módulo basado en la intercomprensión? Durante un año, los estudiantes aprenderían, a partir de un mínimo de latín, las diferentes lenguas — francés, español e italiano en Brasil; italiano, francés y portugués en Colombia; etc.-, entonces podrían elegir una de estas lenguas al tiempo que tendrían algunos conocimientos de las otras.

Otro ejemplo muy distinto tiene que ver con lo que he llamado la diversidad vertical que tiene en cuenta un país oficialmente francófono y otro lusófono: Haití y Cabo Verde. Estos dos países tienen un perfil sociolingüístico similar: una lengua oficial (francés o portugués) que se habla poco como L1 y un creole que todos hablan; aunque el creole haitiano es una lengua cooficial, algo que los caboverdianos quisieran lograr con su propio creole. Por consiguiente, los caboverdianos podrían ir a ver qué hacen los haitianos, evaluar su política y, posiblemente, inspirarse en ella. Allí habría una cooperación romance, si se puede decir, además de una cooperación Sur-Sur, que no solo tendría en cuenta la defensa de las lenguas dominantes, sino también la promoción de las lenguas del pueblo.

Solo he presentado algunos ejemplos de lo que podría ser una geopolítica de las lenguas romances y las políticas lingüísticas entre estos espacios, estas xfonías. Para profundizar sobre este tema, se puede consultar los textos en francés Géopolitique de la langue française (Giblin, 2007) y Langues romanes: un milliard de locuteurs (Éditions CNRS, 2016). 


\section{Referencias}

Blanche-Benveniste, C. y Valli, A. (1997). L'intercompréhension: le cas des langues romanes. Le français dans le monde, Janvier, (22).10-152.

Calhoun, D. (1998). Britannica book of the year. Encyclopaedia Britannica.

Calvet, L.-J. (2002). Le marché au langues : essai de politologie linguistique sur la mondialisation. Plon.

Calvet, L.-J. (2007). Approche sociolinguistique de l'avenir du français dans le monde. Hérodote, 126(3), 153. https://doi.org/10.3917/her.126.0153

Calvet, L.-J. (2018). Pour une géopolitique des langues romanes. Matices en Lenguas Extranjeras (MALE), 12, 9-22. https://revistas.unal.edu.co/index.php/male/article/download/82871/72675/442689

Calvet, L.-J. y Calvet, A. (2016). Les langues romanes dans les universités. Hermes, La Revue, 2(75), 52-59. https://www.cairn.info/revue-hermes-la-revue-2016-2-page-52.htm

Conseil de l'Europe. (1997). Convention sur la reconnaissance des qualifications relatives à l'enseignement supérieur dans la Région Européenne. Unesco. https://www.coe.int/fr/web/conventions/ full-list?module $=$ treaty-detail\&treatynum $=165$

Dabène, L. (2003). De Galatea à Galanet. Lidil (28), 23-29.

Eberhard, D., Simons, G. y Fennig, C. (Éds.). (2019). Welcome to the 22nd edition. Ethnologue (éd. 22). SIL International. https://www.ethnologue.com/ethnoblog/gary-simons/welcome-22nd-edition

Éditions CNRS. (2016). Langues romanes: un milliard de locuteurs [número temático]. Hermès, 75. http://documents.irevues.inist.fr/handle/2042/61425

Giblin, B. (2007). Géopolitique de la langue française. Hérodote, 126(3), 3. https://www.herodote.org/ spip.php?rubrique38

Hoyos, G., Calvet, L.-J., Sauvé, P., y García Canclini, N. (2001). Actes du Colloque "Trois espaces linguistiques face aux défis de la mondialisation". http://www.mondialisations.org/medias/pdf/ Actes3EL_1_P1.pdf

Organisation Internationale de la Francophonie (OIF). (2001, 14-15 de junio). III Conférence ministérielle de la fracophonie sur la culture. Déclaration et plan d'action de Cotonou [conferencia] Cotonú, Benín. https://www.francophonie.org/sites/default/files/2021-01/Conf_minis_Culture_Cotonou-2001.pdf

RFI. (2009, 12 de marzo). Mapa de lenguas con estatus de lenguas oficiales. En RFI, La langue française dans le monde. RFI. http://www1.rfi.fr/actufr/articles/111/article_79172.asp

Statistiques mondiales. (2014). Langues officielles dans le monde. https://bit.ly/2UyxVFY

wikiLF. (2017). Baromètre Calvet des langues du monde. http://www.wikilf.culture.fr/barometre2017/ index.php 